\title{
CLASS OF BOUNDED OPERATORS ASSOCIATED WITH AN ATOMIC SYSTEM
}

\author{
P. SAM JOHNSON AND G. RAMU
}

\begin{abstract}
K$-frames, more general than the ordinary frames, have been introduced by Laura Găvruţa in Hilbert spaces to study atomic systems with respect to a bounded linear operator. Using the frame operator, we find a class of bounded linear operators in which a given Bessel sequence is an atomic system for every member in the class.
\end{abstract}

\section{Introduction}

Frames in Hilbert spaces were introduced by J. Duffin and A.C. Schaffer [1] in 1952, in the context of nonharmonic Fourier series. After a couple of years, in 1986, frames were brought to life by Daubechies, Grossmann and Meyer [2]. Now frames play an important role not only in the theoretics but also in many kinds of applications, and have been widely applied in signal processing [3], sampling theory [4], coding and communications [5] and so on. The notion of $K$-frames has been recently introduced by Laura Găvruţa to study the atomic systems with respect to a bounded linear operator $K$ in Hilbert spaces. It is known that $K$-frames are more general than ordinary frames, and many properties for ordinary frames may not hold for $K$-frames. Several methods to construct $K$-frames and the stability of perturbations for the $K$-frames have been discussed in [6]. In this paper, we construct a frame sequence for the closed subspace $R(K)$ (the range of $K$ ) from an atomic system for a closed range operator $K$. In the end, we find a class of bounded linear operators in which a given Bessel sequence is an atomic system for every member in the class.

Throughout the paper, $H$ is a separable Hilbert space. We denote by $\mathscr{B}(H)$ the space of all bounded linear operators on $H$. For $T \in \mathscr{B}(H)$, we denote by $R(T)$ the range of $T$ and $N(T)$ the null space of $T$.

Received February 17, 2014, accepted April 22, 2014.

2010 Mathematics Subject Classification. 42C15.

Key words and phrases. Bessel sequences, frames, atomic systems, $K$-Frames.

Corresponding author: G. Ramu. 


\section{Notations and preliminaries}

Definition 2.1. A family $\left\{f_{i}\right\}_{i=1}^{\infty}$ of vectors in $\mathrm{H}$ is called a Bessel sequence if there exists a constant $B>0$ such that

$$
\sum_{i=1}^{\infty}\left|\left\langle f, f_{i}\right\rangle\right|^{2} \leq B\|f\|^{2}, \text { for all } f \in H .
$$

For a Bessel sequence $\left\{f_{i}\right\}_{i=1}^{\infty}$, an operator $T: \ell_{2} \rightarrow H$ defined by $T\left(\left\{c_{i}\right\}_{i=1}^{\infty}\right)=\sum_{i=1}^{\infty} c_{i} f_{i}$, is bounded. $T$ is called the pre-frame operator or the synthesis operator. The adjoint of $T, T^{*}$ : $H \rightarrow \ell_{2}$ defined by $T^{*} f=\left\{\left\langle f, f_{i}\right\rangle\right\}_{i=1}^{\infty}$ is called the analysis operator. By composing $T$ and $T^{*}$, we obtain the frame operator

$$
S f=T T^{*} f=\sum_{i=1}^{\infty}\left\langle f, f_{i}\right\rangle f_{i}, \text { for } f \in H .
$$

Moreover, for each $f \in H,\langle S f, f\rangle=\sum_{i=1}^{\infty}\left|\left\langle f, f_{i}\right\rangle\right|^{2}, S$ is a bounded positive self-adjoint operator and by Lemma A.6.7 in [7], $S$ has a unique positive square root, denoted by $S^{1 / 2}$.

Definition 2.2. A Bessel sequence $\left\{f_{i}\right\}_{i=1}^{\infty}$ is a frame for $H$ if there is a constant $A>0$ such that

$$
A\|f\|^{2} \leq \sum_{i=1}^{\infty}\left|\left\langle f, f_{i}\right\rangle\right|^{2} \text {, for all } f \in H .
$$

$A$ and $B$ are called the lower and upper frame bounds for the frame, they are not unique.

Definition 2.3 ([8]). Let $K \in \mathscr{B}(H)$. A sequence $\left\{f_{i}\right\}_{i=1}^{\infty}$ in $H$ is called an atomic system for $K$, if the following conditions are satisfied :

1. $\left\{f_{i}\right\}_{i=1}^{\infty}$ is a Bessel sequence;

2. there exixts $c>0$ such that for every $f \in H$ there exists $a_{f}=\left\{a_{i}\right\}_{i=1}^{\infty} \in \ell_{2}$ such that $\left\|a_{f}\right\|_{\ell_{2}} \leq$ $c\|f\|$ and $K f=\sum_{i=1}^{\infty} a_{i} f_{i}$.

Every operator $K \in \mathscr{B}(H)$ has an atomic system. One may ask whether every Bessel sequence $\left\{f_{i}\right\}_{i=1}^{\infty}$ has an operator $K$ which makes $\left\{f_{i}\right\}_{i=1}^{\infty}$ an atomic system for $K$. The answer is in the affirmative by the following proposition.

Proposition 2.4. Let $\left\{f_{i}\right\}_{i=1}^{\infty}$ be a Bessel sequence in $H$. Then $\left\{f_{i}\right\}_{i=1}^{\infty}$ is an atomic system for the frame operator $S$.

Proof. Since $\left\{f_{i}\right\}_{i=1}^{\infty}$ is a Bessel sequence in $H$, the frame operator $S$ defined as in (2.2), is bounded on $H$. Let $a_{f}=\left\{a_{i}\right\}_{i=1}^{\infty}=\left\{\left\langle f, f_{i}\right\rangle\right\}_{i=1}^{\infty} \in \ell_{2}$. Now

$$
\left\|a_{f}\right\|_{\ell_{2}}^{2}=\left\|\left\{\left\langle f, f_{i}\right\rangle\right\}_{i=1}^{\infty}\right\|_{\ell_{2}}^{2}=\sum_{i=1}^{\infty}\left|\left\langle f, f_{i}\right\rangle\right|^{2} \leq B\|f\|^{2} .
$$

As $\left\|a_{f}\right\|_{\ell_{2}} \leq \sqrt{B}\|f\|$ for each $f \in H$ and $\left\{f_{i}\right\}_{i=1}^{\infty}$ is a Bessel sequence, $\left\{f_{i}\right\}_{i=1}^{\infty}$ is an atomic system for the frame operator $S$. 
Definition 2.5. [8] Let $K \in \mathscr{B}(H)$. A sequence $\left\{f_{i}\right\}_{i=1}^{\infty}$ in $H$ is called a $K$-frame for $H$ if there exist constants $A, B>0$ such that

$$
A\left\|K^{*} f\right\|^{2} \leq \sum_{i=1}^{\infty}\left|\left\langle f, f_{i}\right\rangle\right|^{2} \leq B\|f\|^{2}, \text { for all } f \in H .
$$

We call $A, B$ the lower and upper frame bounds for the $K$-frame $\left\{f_{i}\right\}_{i=1}^{\infty}$ respectively.

Definition 2.6. Let $\left\{f_{i}\right\}_{i=1}^{\infty}$ be a sequence in $H$. We say that $\left\{f_{i}\right\}_{i=1}^{\infty}$ is a frame sequence if it is a frame for the closed subspace $\overline{\operatorname{span}}\left\{f_{i}\right\}_{i=1}^{\infty}$ of $H$.

Definition 2.7 ([9]). Let $H$ be a Hilbert space, and suppose that $E \in \mathscr{B}(H)$ has a closed range. Then there exists an operator $E^{\dagger} \in \mathscr{B}(H)$ for which

$$
N\left(E^{\dagger}\right)=R(E)^{\perp}, \quad R\left(E^{\dagger}\right)=N(E)^{\perp}, \quad E E^{\dagger} y=y, \quad y \in R(E) .
$$

We call the operator $E^{\dagger}$ the pseudo-inverse of $E$. This operator is uniquely determined by these properties. In fact, if $E$ is invertible, then we have $E^{-1}=E^{\dagger}$.

Definition 2.8 ([10]). Assume that $S, K \in \mathscr{B}(H)$. Then $S$ majorizes $K$ if there exists $M>0$ such that $\|K x\| \leq M\|S x\|$ for all $x \in H$.

Theorem 2.9 (Douglas' majorization theorem [10]). Let $H$ be a Hilbert space and $S, K \in \mathscr{B}(H)$. Then the following are equivalent:

1. $R(K) \subseteq R(S)$;

2. $K K^{*} \leq \lambda^{2} S S^{*}$ for some $\lambda \geq 0$ (i.e., $S^{*}$ majorizes $K^{*}$ );

3. $K=S U$ for some $U \in \mathscr{B}(H)$.

\section{Main results}

Theorem 3.1 ([8]). Let $\left\{f_{i}\right\}_{i=1}^{\infty}$ be a sequence in $H$ and $K \in \mathscr{B}(H)$. Then the following statements are equivalent:

1. $\left\{f_{i}\right\}_{i=1}^{\infty}$ is an atomic system for $K$;

2. $\left\{f_{i}\right\}_{i=1}^{\infty}$ is a $K$-frame for $H$;

3. there exists a Bessel sequence $\left\{g_{i}\right\}_{i=1}^{\infty}$ such that $K f=\sum_{i=1}^{\infty}\left\langle f, g_{i}\right\rangle f_{i}$.

Theorem 3.2. [6] Let $\left\{f_{i}\right\}_{i=1}^{\infty}$ be a Bessel sequence in $H$ and $K \in \mathscr{B}(H)$. Then $\left\{f_{i}\right\}_{i=1}^{\infty}$ is a $K$ frame for $H$ if and only if there exists $A>0$ such that $S \geq A K K^{*}$, where $S$ is the frame operator for $\left\{f_{i}\right\}_{i=1}^{\infty}$. 
Each atomic system is associated with a bounded operator on $H$. We analyse a class of operators in $\mathscr{B}(H)$ associated with a given atomic system.

Theorem 3.3. Let $K_{1}, K_{2} \in \mathscr{B}(H)$. If $\left\{f_{i}\right\}_{i=1}^{\infty}$ is an atomic system for $K_{1}$ and $K_{2}$, and $\alpha$, $\beta$ are scalars, then $\left\{f_{i}\right\}_{i=1}^{\infty}$ is an atomic system for $\alpha K_{1}+\beta K_{2}$ and $K_{1} K_{2}$.

Proof. It is given that $\left\{f_{i}\right\}_{i=1}^{\infty}$ is an atomic system for $K_{1}$ and $K_{2}$, then there are positive constants $A_{n}, B_{n}>0(n=1,2)$ such that

$$
A_{n}\left\|K_{n}^{*} f\right\|^{2} \leq \sum_{i=1}^{\infty}\left|\left\langle f, f_{i}\right\rangle\right|^{2} \leq B_{n}\|f\|^{2} \text {, for all } f \in H .
$$

By simple calculations, we have

$$
\frac{A_{1} A_{2}}{A_{2}|\alpha|^{2}+A_{1}|\beta|^{2}}\left\|\left(\alpha K_{1}+\beta K_{2}\right)^{*} f\right\|^{2} \leq \sum_{i=1}^{\infty}\left|\left\langle f, f_{i}\right\rangle\right|^{2} .
$$

Hence $\left\{f_{i}\right\}_{i=1}^{\infty}$ satisfies the lower frame condition. And from inequalities (3.1), we get

$$
\sum_{i=1}^{\infty}\left|\left\langle f, f_{i}\right\rangle\right|^{2} \leq\left(\frac{B_{1}+B_{2}}{2}\right)\|f\|^{2}, \text { for all } f \in H .
$$

Therefore $\left\{f_{i}\right\}_{i=1}^{\infty}$ is an atomic system for $\alpha K_{1}+\beta K_{2}$.

Now for each $f \in H$, we have $\left\|\left(K_{1} K_{2}\right)^{*} f\right\|^{2}=\left\|K_{2}{ }^{*} K_{1}{ }^{*} f\right\|^{2} \leq\left\|K_{2}{ }^{*}\right\|^{2}\left\|K_{1}{ }^{*} f\right\|^{2}$. Since $\left\{f_{i}\right\}_{i=1}^{\infty}$ is an atomic system for $K_{1}$,

$$
\frac{\left\|\left(K_{1} K_{2}\right)^{*} f\right\|^{2}}{\left\|K_{2}{ }^{*}\right\|^{2}} \leq\left\|K_{1}{ }^{*} f\right\|^{2} \leq \frac{1}{A_{1}} \sum_{i=1}^{\infty}\left|\left\langle f, f_{i}\right\rangle\right|^{2} \leq \frac{B_{1}}{A_{1}}\|f\|^{2} .
$$

This implies that $\frac{A_{1}}{\left\|K_{2}{ }^{*}\right\|^{2}}\left\|\left(K_{1} K_{2}\right)^{*} f\right\|^{2} \leq \sum_{i=1}^{\infty}\left|\left\langle f, f_{i}\right\rangle\right|^{2} \leq B_{1}\|f\|^{2}$, for all $f \in H$. Therefore $\left\{f_{i}\right\}_{i=1}^{\infty}$ is an atomic system for $K_{1} K_{2}$.

Corollary 3.4. If $\left\{f_{i}\right\}_{i=1}^{\infty}$ is an atomic system for $\mathscr{A}$, where $\mathscr{A} \subseteq \mathscr{B}(H)$, then $\left\{f_{i}\right\}_{i=1}^{\infty}$ is an atomic system for any operator in the subalgebra generated by $\mathscr{A}$.

Corollary 3.5. If $\left\{f_{i}\right\}_{i=1}^{\infty}$ is an atomic system for a normal operator $K$, then $\left\{f_{i}\right\}_{i=1}^{\infty}$ is an atomic system for any operator in the subalgebra generated by $K$ and $K^{*}$.

Theorem 3.6. Let $\left\{f_{i}\right\}_{i=1}^{\infty}$ be an atomic system for a closed range operator $K$ (i.e., $K$ has a closed range). Then there exists a Bessel sequence $\left\{g_{i}\right\}_{i=1}^{\infty}$ such that $\left\{\left(\left.K^{\dagger}\right|_{R(K)}\right)^{*} g_{i}\right\}_{i=1}^{\infty}$ is a frame sequence for $R(K)$.

Proof. As $\left\{f_{i}\right\}_{i=1}^{\infty}$ is an atomic system, by Theorem 3.1, there exists a Bessel sequence $\left\{g_{i}\right\}_{i=1}^{\infty}$ such that $K f=\sum_{i=1}^{\infty}\left\langle f, g_{i}\right\rangle f_{i}$. Since $\left\{g_{i}\right\}_{i=1}^{\infty}$ is a Bessel sequence, there exists $B>0$ such that

$$
\sum_{i=1}^{\infty}\left|\left\langle f, g_{i}\right\rangle\right|^{2} \leq B\|f\|^{2}, \text { for every } f \in H .
$$


Hence

$$
\sum_{i=1}^{\infty}\left|\left\langle f, K^{\dagger^{*}} g_{i}\right\rangle\right|^{2} \leq D\|f\|^{2} \text {, where } D=B\left\|K^{\dagger}\right\|^{2} .
$$

Using the definition of pseudo-inverse and (3) of Theorem 3.1, for any $f \in R(K)$,

$$
f=K K^{\dagger} f=\sum_{i=1}^{\infty}\left\langle K^{\dagger} f, g_{i}\right\rangle f_{i}=\sum_{i=1}^{\infty}\left\langle f, K^{\dagger^{*}} g_{i}\right\rangle f_{i} .
$$

Also

$$
\|f\|^{4}=|\langle f, f\rangle|^{2}=\left|\left\langle f, \sum_{i=1}^{\infty}\left\langle f, K^{\dagger^{*}} g_{i}\right\rangle f_{i}\right\rangle\right|^{2} \leq \sum_{i=1}^{\infty}\left|\left\langle f, K^{\dagger^{*}} g_{i}\right\rangle\right|^{2} B\|f\|^{2} .
$$

Therefore $\frac{1}{B}\|f\|^{2} \leq \sum_{i=1}^{\infty}\left|\left\langle f, K^{\dagger^{*}} g_{i}\right\rangle\right|^{2}$, for all $f \in R(K)$. Thus $\left\{\left(\left.K^{\dagger}\right|_{R(K)}\right)^{*} g_{i}\right\}_{i=1}^{\infty}$ is a frame sequence for $R(K)$.

The following example illustrates that a Bessel sequence $\left\{f_{i}\right\}_{i=1}^{\infty}$ is an atomic system for an operator $K$ but it is not the same for other operator $L$.

Example 3.7. Let $H=\mathbb{C}^{3}$ and $\left\{e_{1}, e_{2}, e_{3}\right\}$ be an orthonormal basis for $H$. Define $K: H \rightarrow H$ by $K e_{1}=e_{1}, K e_{2}=e_{1}, K e_{3}=e_{2}$. Then $\left\{f_{i}\right\}_{i=1}^{3}=\left\{e_{1}, e_{1}, e_{2}\right\}$ is a $K$-frame for $H$. The frame operator is $S=\left(\begin{array}{lll}2 & 0 & 0 \\ 0 & 1 & 0 \\ 0 & 0 & 0\end{array}\right)$ and its square root is $S^{1 / 2}=\left(\begin{array}{ccc}\sqrt{2} & 0 & 0 \\ 0 & 1 & 0 \\ 0 & 0 & 0\end{array}\right)$. Let $L=\left(\begin{array}{lll}2 & 0 & 0 \\ 0 & 1 & 0 \\ 0 & 1 & 1\end{array}\right)$ and $f=e_{3} \in H$. Then $\sum_{i=1}^{3}\left|\left\langle f, f_{i}\right\rangle\right|^{2}=0$ and $\left\|L^{*} f\right\|^{2}=4$. Hence $\left\{f_{i}\right\}_{i=1}^{3}$ is not a $L$-frame for $H$.

Theorem 3.8. Let $\left\{f_{i}\right\}_{i=1}^{\infty}$ be a Bessel sequence in $H$. Then $\left\{f_{i}\right\}_{i=1}^{\infty}$ is a K-frame for $H$ if and only if $K=S^{1 / 2} T$, for some $T \in \mathscr{B}(H)$.

Proof. Suppose $\left\{f_{i}\right\}_{i=1}^{\infty}$ is a $K$-frame, by Theorem 3.2, there exists $A>0$ such that

$$
A K K^{*} \leq S^{1 / 2} S^{1 / 2^{*}}
$$

Then by definition of inner product, for each $f \in H,\left\|K^{*} f\right\|^{2} \leq A^{-1}\left\|S^{1 / 2} f\right\|^{2}$. Therefore $S^{1 / 2}$ majorizes $K^{*}$. By Douglas' majorization theorem, $K=S^{1 / 2} T$, for some $T \in \mathscr{B}(H)$.

On the other hand, let $K=S^{1 / 2} T$ for some $T \in \mathscr{B}(H)$. Then by Douglas' majorization theorem, $S^{1 / 2}$ majorizes $K^{*}$. Then there is a positive $A$ such that

$$
\left\|K^{*} f\right\| \leq A\left\|S^{1 / 2} f\right\|, \text { for all } f \in H
$$

which implies that $K K^{*} \leq A^{2} S$. Hence by Theorem 3.2, $\left\{f_{i}\right\}_{i=1}^{\infty}$ is a $K$-frame for $H$.

Remark 3.9. In the above example, the operator $L$ is not of the form $S^{1 / 2} T$, for any operator $T \in \mathscr{B}(H)$, because $L$ has a column which is not a linear combination of columns of $S^{1 / 2}$. 


\section{Acknowledgement}

The present work of first author was partially supported by National Board for Higher Mathematics (NBHM), Ministry of Atomic Energy, Government of India (Reference No.2/48 (16)/2012/NBHM(R.P.)/R\&D 11/9133). The authors would like to thank the anonymous referee for valuable comments and suggestions to improve the quality of the paper.

\section{References}

[1] R. J. Duffin and A. C. Schaeffer, A class of nonharmonic Fourier series, Trans. Amer. Math. Soc., 72(1952), 341-366.

[2] I. Daubechies, A. Grossmann and Y. Meyer, Painless nonorthogonal expansions, J. Math. Phys., 27(1986), 1271-1283.

[3] P. Ferreira, Mathematics for multimedia signal processing II: Discrete finite frames and signal reconstruction, Byrnes, J.S. (ed.) 17(1999), 35-54.

[4] Y. C. Eldar, Sampling with arbitrary sampling and reconstruction spaces and oblique dual frame vectors, J. Fourier Anal. Appl., 9(2003), 77-96.

[5] T. Strohmer and R. W. Heath, Jr., Grassmannian frames with applications to coding and communication, Appl. Comput. Harmon. Anal., 14(2003), 257-275.

[6] X. Xiao, Y. Zhu, L. Gǎvruţa, Some properties of K-frames in Hilbert spaces, Results Math., 63 (2013), $1243-1255$.

[7] O. Christensen, An introduction to frames and Riesz bases, Applied and Numerical Harmonic Analysis, Birkhäuser Boston Inc., Boston, MA, 2003.

[8] L. Găvruţa, Frames for operators, Appl. Comput. Harmon. Anal., 32(2012), 139-144.

[9] P. G. Casazza, The art of frame theory, Taiwanese J. Math., 4(2000), 129-201.

[10] R. G. Douglas, On majorization, factorization, and range inclusion of operators on Hilbert space, Proc. Amer. Math. Soc., 17(1966), 413-415.

Department of Mathematical and Computational Sciences, National Institute of Technology Karnataka, Surathkal, Mangalore 575 025, India.

E-mail: sam@nitk.ac.in

Department of Mathematical and Computational Sciences, National Institute of Technology Karnataka, Surathkal, Mangalore 575 025, India.

E-mail: ramu.geddavalasa@gmail.com 